\title{
Pirólisis catalítica de cáscara de maní para la obtención de productos químicos de interés
}

\section{Catalytic pyrolysis of peanut shells to obtain value added products.}

\section{Doctorando:}

\section{Fermanelli, Carla Soledad}

CITeQ-CONICET-UTN

cfermanelli@frc.utn.edu.ar

\section{Director/es:}

\section{Liliana Pierella}

\section{Clara Saux}

\section{Resumen}

Se estudió la pirólisis catalítica de cáscaras de maní (Arachis hipogaea) en un reactor de lecho fijo que integra los procesos de pirólisis y craqueo catalítico. Se analizó el efecto de los catalizadores sobre la composición del bio-oil, incorporando diferentes cantidades de Níquel a zeolitas ZSM-11, por el método de impregnación húmeda. Las experiencias se realizaron con cargas de metal en un rango desde $1 \%$ a $10 \% \mathrm{p} / \mathrm{p}$. Estos materiales fueron exhaustivamente caracterizados por Difracción de Rayos X, Reducción a Temperatura Programada TPR y Análisis Termogravimétrico.

Al evaluar la composición del bio-oil generado, se encontró que aumenta la selectividad hacia algunos hidrocarburos aromáticos y al dehídos de interés. Los mejores resultados se obtuvieron cuando el contenido del metal fue del $3 \% \mathrm{p} / \mathrm{p}$, disminuyendo para cargas menores y mayores..

Palabras claves: Pirólisis Catalítica, Cáscaras de Maní, Bio-oil, Ni-ZSM-11.

\begin{abstract}
Catalytic pyrolysis of peanut shells (Arachis hipogaea) were studied in a fixed-bed reactor, which integrates both processes, pyrolysis and catalytic cracking. The effect of the catalytic material over bio-oil composition was studied, introducing Nickel by wet impregnation method in ZSM-11 zeolites. The experiments were done with different loading of metal, ranging from 1 to $10 \mathrm{wt} \%$. These catalysts were thoroughly characterized by X-Ray Diffraction, Temperature Programmed Reduction and Thermogravimetric Analysis.

Evaluating bio-oil composition, it was found that some desired aromatic hydrocarbons and aldehydes selectivities were improved. Best results were obtained when $3 \mathrm{wt} \%$ of the metal was loaded, reducing for lower and higher contents.
\end{abstract}

Keywords: Catalytic Pyrolysis, Peanut Shell, Bio-oil, Ni-ZSM-11.

\section{Introducción}


Uno de los desafíos que se nos presenta en la actualidad es el de alcanzar un desarrollo sustentable, es decir, hacer compatible el desarrollo económico con la calidad del medio ambiente. En este sentido se plantea la obtención de moléculas plataforma para la industria química fina a partir de fuentes renovables (biomasa) y, particularmente, residuos de un proceso productivo, como es el caso de las cáscaras de maní. En la provincia de Córdoba se cultivan anualmente casi 1 millón de toneladas de maní (95\% de la producción nacional) (MAGYP, 2019) y de este valor aproximadamente el $25 \%$ corresponde a su cáscara, que es separada en las plantas seleccionadoras y procesadoras de esta legumbre, transformándose en el desecho más abundante de la industrialización de la misma (Bolsa de Comercio de Córdoba, 2006). En la actualidad parte de este desecho se quema en calderas para la obtención de energía, pero un volumen importante no tiene un destino económicamente rentable y es quemado a cielo abierto, con el consecuente impacto ambiental. De manera que la disponibilidad de cáscaras de maní es, en la provincia de Córdoba, abundante, concentrada y sin valor de mercado (Gatani, Argüello and Sesín, 2010).

Nuestra propuesta consiste en la obtención de un bio-oil con una composición rica en moléculas de interés industrial a partir de un proceso de pirólisis-craqueo combinados en un reactor tubular mediante el empleo de zeolitas ZSM-11 modificadas con Níquel.

\section{Experimental}

Preparación y caracterización de los catalizadores

Las zeolitas microporosas ZSM-11 se prepararon por síntesis hidrotérmica empleando aluminato de sodio, sílice, hidróxido de tetrabutilamonio como agente director de estructura y agua destilada en autoclave a $150^{\circ} \mathrm{C}$ y presión autogenerada durante 10 días. Posteriormente, el material fue lavado y secado en estufa. Luego se desorbió en corriente de $\mathrm{N}_{2}$ y temperatura programada hasta $500{ }^{\circ} \mathrm{C}$ durante $8 \mathrm{~h}$ y finalmente calcinada con el mismo programa térmico. Para obtener la forma $\mathrm{NH}_{4}$-zeolita se realizó un intercambio a $80^{\circ} \mathrm{C}$ con una solución $1 \mathrm{M}$ de cloruro de amonio sobre este material. A partir de esta expresión se desorbió en corriente de $\mathrm{N}_{2}$ para obtener la forma protónica H-ZSM-11 o se impregnó por vía húmeda a partir de una solución acuosa de $\mathrm{NiCl}_{2} .6 \mathrm{H}_{2} \mathrm{O}$ incorporando $1 \%$, 3\% y $10 \%$ p/p de Níquel para la obtención de las formas $\mathrm{Ni}(1)-\mathrm{ZSM}-11, \mathrm{Ni}(3)-\mathrm{ZSM}$ 11 y Ni(10)-ZSM-11, denominadas en este trabajo como $\mathrm{Ni}$ (1), Ni(3) y Ni(10) respectivamente. A la forma protónica se la denominó $\mathrm{H}$. Por último fueron desorbidas a $500^{\circ} \mathrm{C}$ en corriente de $\mathrm{N}_{2}$ y luego calcinadas en atmósfera oxidante por $10 \mathrm{~h}$ a la misma temperatura.

El área superficial de los materiales frescos (inmediatamente después de su síntesis y modificación) y usados (luego de la reacción, sin tratamientos posteriores) se determinó por el método Brunauer-Emmet-Teller (BET) en un equipo Pulse Chemisorb 270 de Micromeritics con absorción de $\mathrm{N}_{2}$ a $77 \mathrm{~K}$. El comportamiento reductivo se estudió mediante la técnica de Reducción a Temperatura Programada de $\mathrm{H}_{2}\left(\mathrm{H}_{2}\right.$-TPR) en un equipo Chemisorb 2720, también de Micromeritics.

La estructura cristalina se analizó a través de Difracción de Rayos X (XRD) de polvos en un difractómetro Philips PW 3020 empleando radiación CuKa de longitud de onda $0.15418 \mathrm{~nm}$. Los datos de difracción se recogieron entre $2 \theta=5-60^{\circ}$, a interval os de 0.10 y velocidad de 20 por $\mathrm{min}$.

\section{Acondicionamiento y caracterización de la biomasa}

La cáscara de maní provista por la empresa Lorenzatti, Ruetsch y Cia., Ticino, Córdoba, Argentina, pasó por un proceso de lavado con agua, secado en estufa a 105 ㄷ C hasta peso constante, molido y tamizado para lograr un tamaño de partícula de diámetro inferior a $3.35 \mathrm{~mm}$ (ASTM E-11/95). El lavado se realiza con el fin de quitar metales que pudieran estar adheridos a la superficie, y que generan efecto depresor sobreel rendimiento del biooil (Oudenhoven, Westerhof and Kersten, 2015). El molido del material reduce los problemas asociados a la difusión de calor en sólidos por mayores tamaños de partículas (Bridgwater, 2012). De acuerdo a Dobele y 
colaboradores (Dobele et al. , 2007) la eliminación del agua en la biomasa inicial disminuyeel contenido de agua, ácidos y ésteres en el bio-oil, y genera un aumento en el rendimiento de productos químicos de interés.

El comportamiento térmico de la biomasa se estudió por termogravimetría en atmósfera inerte, con una termobalanza modelo TGA/SDTA851e/SF $/ 1100^{\circ} \mathrm{C}$ Mettler Toledo. El material de referencia fue a-alúmina ( $\alpha$ $\mathrm{Al}_{2} \mathrm{O}_{3}$ ) en polvo marca Shimadzu.

En relación a la composición, datos publicados (Gurevich Messina, Bonelli and Cukierman, 2015) indican que la cáscara de maní posee $30.9 \%$ de lignina, $54.6 \%$ de celulosa y $14.5 \%$ de hemicelulosa (en base a FND libre de cenizas). El análisis proximal revela un contenido de humedad del $6.5 \%$, volátiles $68.8 \%$, ceniza $5.5 \%$, carbono fijo $19.2 \%$.

El análisis elemental de la biomasa se realizó con un equipo CHN628 Series, Sulfur Add-On Module and TruSpec Micro Oxygen Add-On Module Elemental Determinators marca LECO. Por otra parte, se complementó la determinación elemental por ICP en un equipo ICP-OPTIMA 2100.DV.Perkin Elmer.

Pirólisis catalítica

La pirólisis de la biomasa y el upgrade del bio-oil generado se real izaron simultáneamenteen un reactor tubular de vidrio de lecho fijo. Resumidamente, dentro del reactor se colocó $1 \mathrm{~g}$ de biomasa en una canasta de vidrio de base porosa sobre un lecho conformado por $1 \mathrm{~g}$ de catal izador mezclado con $7 \mathrm{~g}$ de cuarzo molido. Para estudiar la reutilización y la resistencia a la desactivación del catalizador, se realizaron seis reacciones consecutivas sin regeneración, denominadas ciclo $\mathrm{X}$, donde $\mathrm{X}$ indica el número de usos del material sin tratar.

Los productos líquidos se colectaron en una trampa sumergida en un baño salino a $-15{ }^{\circ} \mathrm{C}$. La composición química de los mismos se evaluó en un Cromatógrafo Perkin Elmer Clarus 500 con detector FID y una columna capilar modelo ZB-1 de $30 \mathrm{~m}$ de longitud y $0,53 \mathrm{~mm}$ de diámetro. Se usó un flujo constante de $\mathrm{N}_{2}$ de $1 \mathrm{ml} / \mathrm{min}$ como gas portador. La identificación de los compuestos se realizó por CG-MS, en un cromatógrafo Shimadzu QP 5050 GC-17 A, con una columna HP-5 de 25 m de largo y 0,2 mm de diámetro interno, con He como gas portador y mediante la utilización de patrones. La base de datos NIST fue usada como referencia para determinar los químicos presentes.

Estudios propios determinaron que la temperatura a la cual la pirólisis de la cáscara de maní genera el mayor rendimiento de líquidos es a $500^{\circ} \mathrm{C}$, lo cual también fue demostrado por otros autores con la misma fuente de biomasa (Gurevich Messina, Bonelli and Cukierman, 2015) o con otras (Aysu and Küçük, 2014). El reactor se colocó en el horno una vez al canzada la temperatura de reacción bajo flujo de $\mathrm{N}_{2}(60 \mathrm{ml} / \mathrm{min}$ ) durante $10 \mathrm{~min}$.

El contenido de coque sobre el catalizador usado, luego de la reacción, se determinó por termogravimetría en el equipo previamente descripto. La muestra se colocó en atmósfera oxidante, con un flujo deaire de $75 \mathrm{ml} / \mathrm{min}$, rampa de calentamiento de $10^{\circ} \mathrm{C} / \mathrm{min}$ hasta $600^{\circ} \mathrm{C}$ y mantenida a esa temperatura por $30 \mathrm{~min}$. El cálculo para obtener la cantidad de coque se realizó asumiendo que a la temperatura de $100{ }^{\circ} \mathrm{C}$ la muestra ya estaba seca, y todo el coque se había consumido a los $600^{\circ} \mathrm{C}$. La Eq. (1) se usó para cal cular la cantidad relativa de coque (Aho et al., 2008).

El residuo sólido remanente (carbón) se cuantificó por diferencia de pesada de la canasta antes y después de la reacción. De igual forma se calculó el rendimiento a bio-oil, pesando la trampa de líquidos antes y después de la corrida. Para completar el balance de masas, el rendimiento a productos gaseosos se estimó por diferencia entre producto sólido, líquido y coque, y la biomasa inicial (1g). Las ecuaciones Eq. (2), (3) y (4) se usaron para calcular los rendimientos de los productos.

$$
\begin{aligned}
& \text { Coque }(\% p / p)=\frac{m 100^{\circ} \mathrm{C}-m 600^{\circ} \mathrm{C}}{m 600^{\circ} \mathrm{C}} * 100 \\
& \text { Bio }- \text { oil }(\% p / p)=\frac{\text { W bio-oil }}{\text { W biomasa }} * 100
\end{aligned}
$$


Donde $\mathrm{m}_{100^{\circ} \mathrm{C}}$ es la masa del catalizador a los $100^{\circ} \mathrm{C}, \mathrm{m}_{600^{\circ} \mathrm{C}}$ es la masa del catalizador a los $600{ }^{\circ} \mathrm{C}$, W $\mathrm{W}_{\text {biomasa }}$ es la masa inicial de la biomasa, $W_{\text {carbón }}$ es el peso del residuo sólido remanente luego de la reacción y $W_{\text {bio-oil }}$ es el peso del producto líquido.

\section{Resultados y discusión}

\section{Caracterización de la biomasa}

El análisis termogravimétrico (TGA) es una técnica comúnmente utilizada para estudiar las reacciones de descomposición de materiales lignocelulósicos (Carrier et al., 2011), al igual que su cinética en procesos pirolíticos y la estabilidad térmica de los mismos (Nsaful et al., 2015). Esa estabilidad, en los polisacáridos provenientes de la biomasa depende de su composición, estructura y de la presencia de compuestos inorgánicos que puedan tener efecto catalítico, disminuyendo la temperatura de conversión (Wang et al., 2015). El análisis térmico (curva dTG y pérdida de masa) de la cáscara de maní lavada se muestra en la Figura 1. Un 3\% de pérdida de masa ocurre hasta $\operatorname{los} 100^{\circ} \mathrm{C}$, que se asigna al contenido de humedad del material.

La temperatura inicial de conversión se observa a los $230{ }^{\circ} \mathrm{C}$, reportada en estudios previos para la hemicelulosa de angiospermas, cuyo componente mayoritario es el xilano (Wang et al., 2015). Mientras que el pico principal en la curva DTG se atribuye a la depolimerización de la celulosa, el secundario es atribuible a la conversión de la lignina y se observa a los $530^{\circ} \mathrm{C}$ con el $97 \%$ de pérdida de masa (Raveendran, Ganesh and Khilar, 1996).

El análisis elemental de la cáscara de maní indicó: Carbono 47.73 \%, Hidrógeno $6.47 \%$, Nitrógeno 1.11 \%, Oxígeno $38.95 \%$. Por otra parte, por ICP se determinó: Potasio $0.16 \%$, Calcio 0.24\%; Hierro 0.021\%, Aluminio $0.022 \%$, Silicio $0.037 \%$.

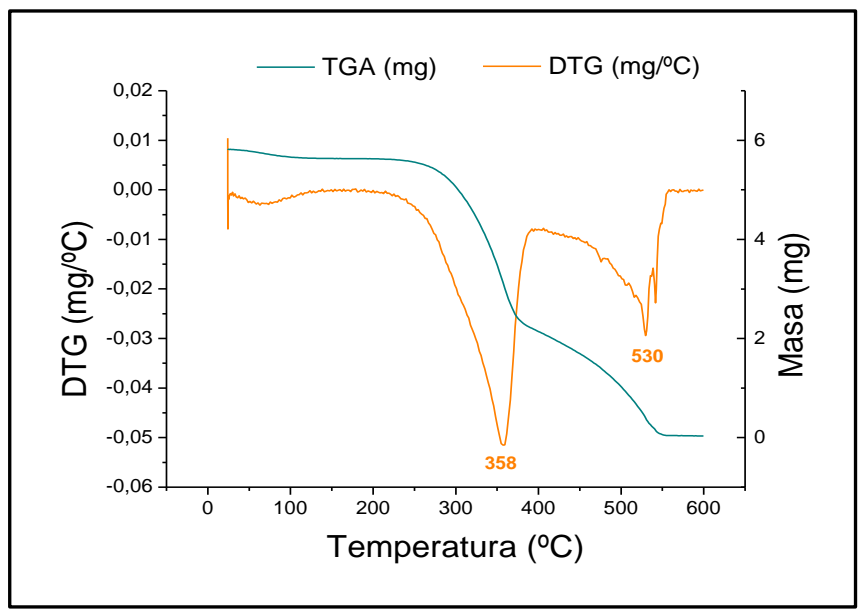

Figura 1: Curvas TG y dTG de la cáscara de maní.

\section{Caracterización de los catalizadores}

En la Tabla 1 se muestra la variación del área superficial de las zeolitas frescas y usadas, al aumentar el contenido de metal en el catalizador. Es conocido que al cargar materiales porosos con metales, el área superficial disminuye debido al bloqueo de los poros y a la sinterización del metal durante la calcinación (Veses et al., 2015; Widayatno et al., 2016), tal como se observa en la Tabla 1. Este efecto se ve más pronunciado a altas cargas de Níquel (10\%).

La Tabla 2 presenta los valores de coque depositados sobre los catalizadores. La zeolita protonada presenta el

mayor valor, observándose una tendencia a disminuir cuando se aumenta la carga del metal en bajas proporciones, tendencia que se revierte cuando el valor alcanza el 10\%, debido posiblemente a los fenómenos de bloqueo y sinterización previamente mencionados. En la Tabla 1 se presentan también los valores de área superficial obtenidos para los materiales usados con una considerable disminución con respecto a sus pares sin uso por efecto de la deposición del coque sobre los poros. 
Tabla 1: Superficie de zeolitas ZSM-11 impregnadas con Níquel

\begin{tabular}{lrrrr}
\hline Carga de metal $(\% \mathrm{p} / \mathrm{p})$ & 0,00 & 1,00 & 3,00 & 10,00 \\
\hline $\mathrm{S}_{\mathrm{BET}}\left(\mathrm{m}^{2} / \mathrm{g}\right)$ fresca & 361 & 331 & 321 & 287 \\
$\mathrm{~S}_{\mathrm{BET}}\left(\mathrm{m}^{2} / \mathrm{g}\right)$ usada & 249 & 260 & 221 & 221 \\
\hline
\end{tabular}

Tabla 2: Contenido relativo de coque $(\% \mathrm{p} / \mathrm{p})$ depositado sobre zeolitas, determinado por TGA

\begin{tabular}{lrrrr} 
Carga de metal $(\% \mathrm{p} / \mathrm{p})$ & 0,00 & 1,00 & 3,00 & 10,00 \\
\hline Coque & 7.23 & 4.71 & 4.44 & 5.94 \\
\hline
\end{tabular}

Los resultados obtenidos por Difracción de Rayos X se muestran en la Figura 2, los cuales confirman la el evada estabilidad de este tipo de materiales considerando que tanto la estructura ZSM-11 como la cristalinidad, no se vieron afectadas por los tratamientos químicos y térmicos a los que fueron sometidos. Se observan los picos característicos de la zeolita y las señales propias del NiO, que aumentan en intensidad al aumentar la carga de Níquel sobre el material catalítico.

Los perfiles de TPR se presentan en la Figura 3. La zona de reducción se observa entre $300{ }^{\circ} \mathrm{C}$ y $500{ }^{\circ} \mathrm{C}$, ya reportado para partículas de $\mathrm{NiO}$ (I wamoto, 2008; Maia et al., 2010). El consumo de $\mathrm{H}_{2}$ sigue la tendencia encontrada por XRD de incremento con el aumento del contenido de metal, por mayor presencia de NiO.
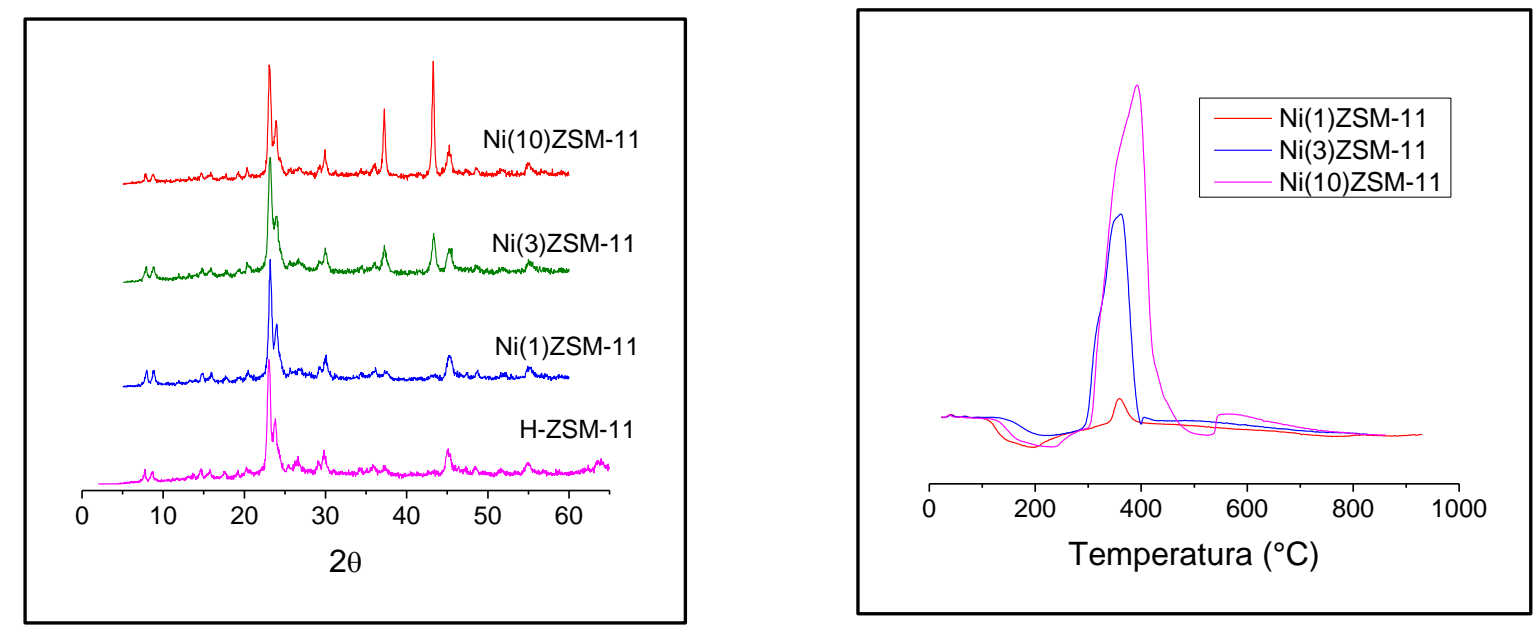

FigIFigura 3: Espectro de TPR de

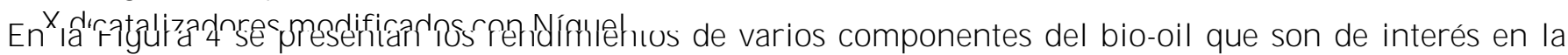
industria química. Los mismos se calcularon como la relación porcentual de ese compuesto frente al total de los componentes del bio-oil. Todos los compuestos, con excepción de furfural, sólo se encontraron como trazas, o no se encontraron cuando la pirólisis se realizó en ausencia de catalizador (térmica). En la figura puede observarse cómo aumenta la selectividad hacia tolueno, m-xileno, cumeno (isopropil benceno) y trimetilbenceno (TMB) cuando la carga de Níquel sobre el catalizador es del 3\%, indicando que una mayor proporción de productos oxigenados son convertidos a hidrocarburos. Este mismo comportamiento se observó en estudios previos para catalizadores impregnados con Cobre (Widayatno et al., 2016). Mayor contenido de Níquel resulta en una disminución en la generación de estos compuestos, incluso por debajo de lo que genera la zeolita $\mathrm{H}$, debido posiblemente a la falta de dispersión de las especies metálicas, por la formación de aglomerados superficiales que dificultarían el contacto entre los sitios activos del catal izador y los reactivos (Widayatno et al., 2016).

El balance de masas de los productos de reacción se presenta en la Figura 5. Los mayores rendimientos a biooil se observan para las pirólisis térmicas. La utilización de catalizadores aumenta el craqueo hacia compuestos 
de menor peso molecular (gases permanentes); sin embargo, las reacciones catalizadas con $\mathrm{Ni}(3)$ y $\mathrm{Ni}(10)$ presentaron rendimientos de bio-oil muy similares a las no catalizadas.

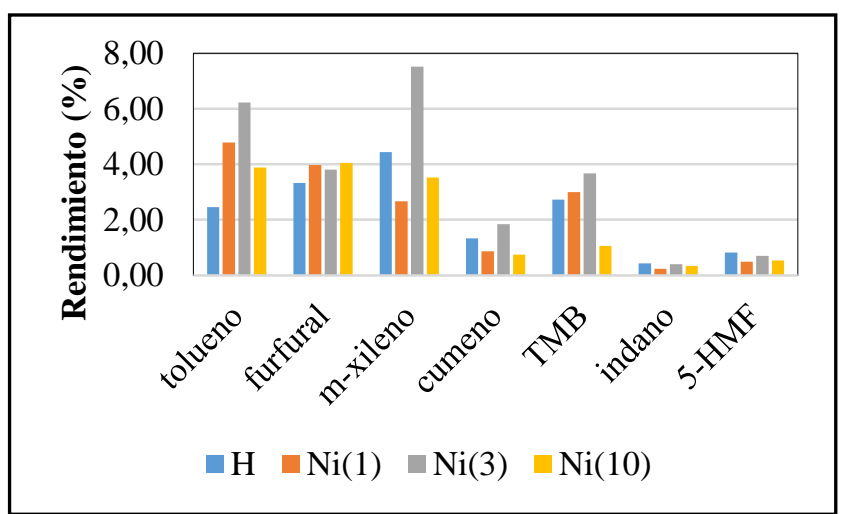

FFigura 5: Balance de masas de productos de hreacción en nresencia.v au..sencia de catalizador.

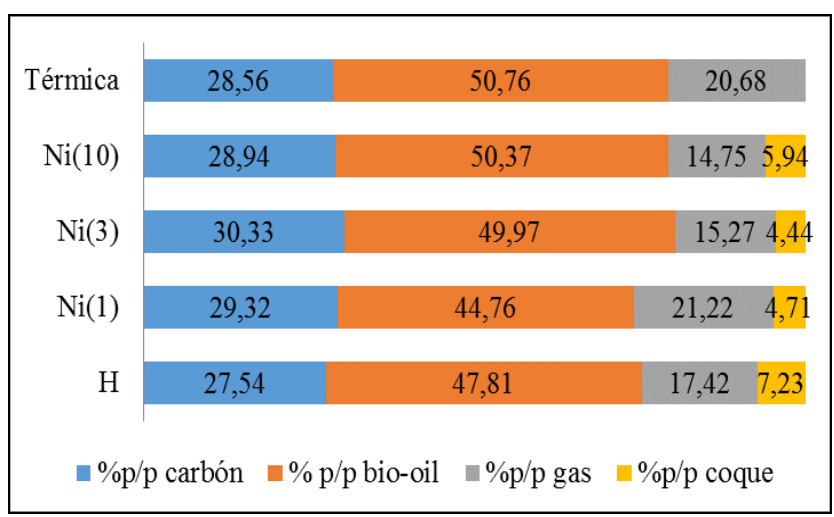

Entre los materiales microporosos, las reacciones que generaron menor producto líquido fueron las catal izadas con $\mathrm{Ni}$ (1), que presentando val ores de coque similares a las otras formas metal-zeolita, evidenciaron los mayores valores de producción de gas, por un mayor craqueo de los vapores de pirólisis.

\section{Conclusiones}

Mediante un proceso combinado de pirólisis-craqueo catalítico se logró mejorar la composición del bio-oil obtenido a partir de un residuo agroindustrial como son las cáscaras de maní .

En base a los resultados obtenidos al caracterizar las zeolitas microporosas modificadas se encontró que la incorporación de Níquel provoca una reducción en el área superficial proporcional al contenido incorporado. Por otra parte, este aumento disminuye la formación de coque sobre el catalizador y mejora la composición de la fracción de productos líquidos.

\section{Agradecimientos}

Las autoras desean expresar su agradecimiento a CONICET (PIP11220130100146CO), UTN (UTN4333), Mincyt Córdoba (PIOdo 2015) y SPU (Universidades Agregando Valor 3454).

\section{Referencias}

Aho, A. et al. (2008) 'Catalytic pyrolysis of woody biomass in a fluidized bed reactor: Influence of the zeolite structure', Fuel, 87(12), pp. 2493-2501. doi: 10.1016/j.fuel.2008.02.015.

Aysu, T. and Küçük, M. M. (2014) 'Biomass pyrolysis in a fixed-bed reactor: Effects of pyrolysis parameters on product yields and characterization of products', Energy, 64, pp. 1002-1025. doi : 10.1016/j.energy.2013.11.053.

Bolsa de Comercio de Córdoba (2006) ‘Encadenamiento productivo del maní', in El Balance dela Economía Argentina 2006 . Una nueva oportunidad. Córdoba: Bolsa de Comercio de Córdoba, pp. 531-548.

Bridgwater, A. V. (2012) 'Review of fast pyrolysis of biomass and product upgrading', Biomass and Bioenergy. Elsevier Ltd, 38, pp. 68-94. doi: 10.1016/j.biombioe.2011.01.048.

Carrier, M. et al. (2011) 'Thermogravimetric analysis as a new method to determine the lignocellulosic composition of 
biomass', Biomass and Bioenergy, 35(1), pp. 298-307. doi: 10.1016/j.biombioe.2010.08.067.

Dobele, G. et al. (2007) 'Fast pyrolysis-effect of wood drying on the yield and properties of bio-oil', BioResources, 2(4), pp. 699-706. doi: 10.15376/biores.2.4.698-706.

Gatani, M., Argüello, R. and Sesín, S. (2010) 'Materiales compuestos de cáscaras de maní y cemento. Influencia de diferentes tratamientos químicos sobre las propiedades mecánicas', M ateriales de Construcción, 60(298), pp. 137-147. doi: $10.3989 / \mathrm{mc} .2010 .46908$.

Gurevich Messina, L. I., Bonelli, P. R. and Cukierman, A. L. (2015) 'Copyrolysis of peanut shells and cassava starch mixtures: Effect of the components proportion', J ournal of Analytical and Applied Pyrolysis. Elsevier B.V., 113, pp. 508517. doi: 10.1016/j.jaap.2015.03.017.

Iwamoto, M. (2008) 'Conversion of ethene to propene on nickel ion-loaded mesoporous silica prepared by the template ion exchange method', Catalysis Surveys from Asia, 12(1), pp. 28-37. doi: 10.1007/s10563-007-9036-y.

MAGYP, M. de A. G. y P. (2019) Estimaciones Agrícolas. Available at:

http://datosestimaciones. magyp.gob.ar/reportes. php?reporte=Estimaciones (Accessed: 2 July 2019).

Maia, A. J. et al. (2010) 'Ni-ZSM-5 catalysts: Detailed characterization of metal sites for proper catalyst design', J ournal of Catalysis. Elsevier Inc., 269(1), pp. 103-109. doi: 10.1016/j.jcat.2009.10.021.

Nsaful, F. et al. (2015) 'Lignocellulose pyrolysis with condensable volatiles quantification by thermogravimetric analysis - Thermal desorption/gas chromatography-mass spectrometry method', J ournal of Analytical and A pplied Pyrolysis, 116, pp. 86-95. doi: 10.1016/j.jaap.2015.10.002.

Oudenhoven, S. R. G., Westerhof, R. J. M. and Kersten, S. R. A. (2015) 'Fast pyrolysis of organic acid leached wood, straw, hay and bagasse: Improved oil and sugar yields', J ournal of Analytical and Applied Pyrolysis. Elsevier B.V., 116, pp. 253-262. doi: 10.1016/j.jaap.2015.09.003.

Raveendran, K., Ganesh, A. and Khilar, K. C. (1996) 'Pyrolysis characteristics of biomass and biomass components', Fuel, 75(8), pp. 987-998. doi: 10.1016/0016-2361(96)00030-0.

Veses, A. et al. (2015) 'Catalytic upgrading of biomass derived pyrolysis vapors over metal-loaded ZSM-5 zeolites: Effect of different metal cations on the bio-oil final properties', M icroporous and M esoporous M aterials. Elsevier Inc., 209, pp. 189-196. doi: 10.1016/j.micromeso.2015.01.012.

Wang, S. et al. (2015) 'Pyrolysis behaviors of four O-acetyl-preserved hemicellul oses isolated from hardwoods and softwoods’, Fuel. Elsevier Ltd, 150, pp. 243-251. doi: 10.1016/j.fuel.2015.02.045.

Widayatno, W. B. et al. (2016) 'Upgrading of bio-oil from biomass pyrolysis over Cu-modified $\beta$-zeolite catalyst with high selectivity and stability', A pplied Catalysis B: Environmental. Elsevier B.V., 186, pp. 166-172. doi:

10.1016/j.apcatb.2016.01.006. 